\title{
Transplantations de neurones fotaux : nouveaux succès mais risque de dérapage
}

Le New England Joumal of Medicine vient de publier une série de trois articles, accompagnés de trois éditoriaux, concernant des expériences de transplantation de neurones fœtaux chez des patients atteints d'une maladie de Parkinson soit idiopathique soit induite par un agent toxique, le MPTP. Il s'agissait très clairement d'une opération à double objectif, politique d'une part, scientifique de l'autre. Par cette publication groupée exceptionnelle, et très directement dans un éditorial de la plume même de son rédacteur en chef [1], le New England Journal of Medicine demandait ainsi au gouvernement américain de lever immédiatement le veto qu'il oppose depuis plus de quatre ans aux expériences portant sur le fœtus humain. On doit, à cette occasion, saluer le courage des deux équipes américaines qui se sont lancées dans cette recherche clinique malgré le veto gouvernemental et en dépit de l'interdiction qui leur était faite d'utiliser des fonds d'État. Pour elles, comme pour les associations - souvent de malades - qui les ont soutenues, cette publication prestigieuse est plus qu'un succès scientifique, c'est la rétribution de plusieurs années de combat d'autant plus difficile qu'il se heurtait non pas à des arguments scientifiques mais à une décision politique.

En ce qui concerne le contenu des articles présentés, deux conclusions majeures peuvent être tirées. Tout d'abord, les transplantations intracérébrales de neurones fœtaux peuvent effectivement corriger des déficits induits par une atteinte neurodégénérative chez l'homme. Ce résultat avait déjà été obtenu, et publié [2-4] (voir aussi $\mathrm{m} / \mathrm{s} n^{\circ} 5$, vol. 8, p. 508), mais l'accroissement du nombre de patients rend la démonstration plus forte. Dans chacun des trois articles, il existe en effet des présentations de cas cliniques qui démontrent une compensation - cits fonctionnels provoqués par l'atteinte des voies dopaminergiques cérébrales (voir $\mathrm{m} / \mathrm{s} n^{\circ} 6$, vol. 8, p. 601). Paradoxalement, toutefois, la publication conjointe des trois articles souligne aussi la difficulté que présente l'évaluation et le suivi des patients parkinsoniens soumis à la procédure de transplantation intracérébrale, difficulté que nous avions déjà évoquée dans une précédente nouvelle de $\mathrm{m} / \mathrm{s}\left(n^{\circ} 6\right.$, vol. 8, p. 601). De ce point de vue, comme l'article de la même équipe évoquée dans la précédente nouvelle, la publication de Widner et al. [5] est un modèle de rigueur scientifique. L'équipe suédoise de Lund présente aujourd'hui non plus des transplants pratiqués chez des patients atteints de maladie de Parkinson idiopathique, mais l'évolution de deux patients intoxiqués au MPTP. Cette substance, qui est un polluant industriel, avait été mélangée à de l'héroïne par des trafiquants. Une dizaine de toxicomanes américains s'étaient ainsi retrouvés en 1981, presque du jour au lendemain, atteints d'une maladie de Parkinson quasiment expérimentale et un grand nombre des neurones dopaminergiques de la substance noire avaient été détruits d'un coup chez cux. Le MPTP est en effet recapté par les cellules dopaminergiques et transformé en un ion toxique pour les mitochondries, le MPP +. Ce sont donc deux de ces patients qui ont été transplantés après plusieurs années d'un syndrome parkinsonien extrêmement fort et mal corrigé par les thérapeutiques usuelles (LDopa et agonistes dopaminergiques). Par rapport aux précédentes transplantations, l'équipe suédoise n'a innové qu'en greffant bilatéralement - en deux temps chirurgicaux rapprochés dans l'ensemble du striatum, c'est-àdire le noyau caudé et le putamen. Pour le reste, et notamment pour le suivi neurologique, qui est le point capital de ces études, l'équipe suédoise a utilisé le même arsenal de tests objectifs détaillés et chiffrés que précédemment, ce qui, de façon fort heureuse, permet de comparer les résultats obtenus chez les différents malades. Ce suivi neurologique rigoureux est directement calqué sur le protocole international élaboré au cours des années passées, le CAPIT (core assessment protocol for intracerebral tramsplantation), ce qui permet en outre de comparer directement les résultats avec ceux obtenus par d'autres groupes qui travaillent dans les mêmes conditions (le nôtre notamment). Les deux courbes de la figure 1 donnent une idée précise du résultat exceptionnellement positif qui a été obtenu. Les mouvements de prono-supination (comptés sur 30 secondes) sont un de ces tests chiffrés qui permettent de tester objectivement la rigidité et l'hypokinésie des patients. Alors que ces mouvements sont très difficiles avant et juste après la transplantation, ils deviennent de plus en plus aisés au fur et à mesure qu'on s'éloigne de l'étape chirurgicale. Dans le même temps, la tomographie par émission de positons révèle une augmentation progressive de la capture de fluoro-Dopa au site de la greffe, indiquant très vraisemblablement une progression de l'efficacité fonctionnelle des neurones transplantés. Cette cohérence parfaite entre les résultats cliniques et au PET-scan, déjà soulignée dans les travaux précédents de cette équipe comme dans le nôtre, est un argument majeur, voire décisif, dans la démonstration de l'effet thérapeutique de la greffe.

Les travaux des deux groupes américains $[6,7]$ ne sont malheureusement pas fondés sur le même protocole ni même sur un ensemble d'épreuves permettant un suivi aussi rigoureux de l'évolution des patients. Il est de ce fait beaucoup plus difficile d'évaluer nombre des résultats cliniques présentés. Ceux-ci dérivent en partie d'études sur 


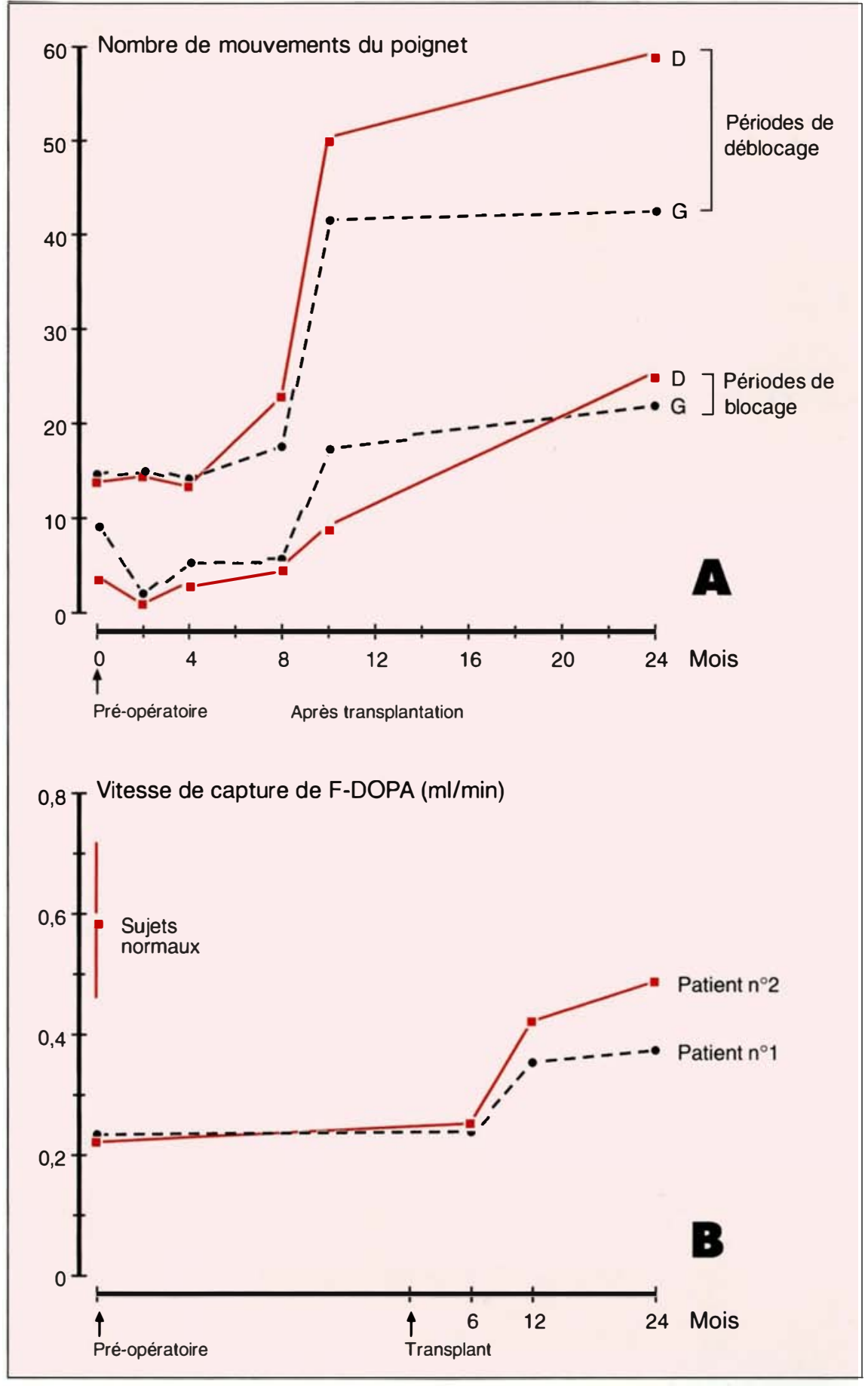

Figure 1. Résultats obtenus lors des tests de prono-supination (nombre de mouvememts en 30 secondes). (A) par le patient 1 et (B) calcul de la vitesse de capture de la fluoro-Dopa à partir des examens à la caméra à émission de positons chez les deux patients de la série suédoise.
" vidéos " qui ne remplacent en aucun cas l'examen pratiqué par un neurologue, a fortiori les épreuves chronométrées. Les symptômes parkinsoniens sont très variables d'un moment à un autre chez le même patient et les prises de vue ont été réalisées sans que soit parfaitement établi l'état du patient, c'est-à-dire sans que l'on puisse savoir clairement s'il était en période de blocage (éventuellement partiel) ou de déblocage (même relatif). L'absence de référence au CAPIT rend pratiquement impossible la comparaison des résultats obtenus dans ces deux études et dans celles d'autres groupes. La difficulté est encore aggravée par l'absence d'examens systématiques par tomographie par émission de positons et le manque de significativité des quelques résultats présentés. Le PET-scan donne des résultats souvent difficiles à apprécier, mais les images produites par le groupe de Denver supportent très mal la comparaison avec celles de l'équipe suédoise. Quant au groupe de Yale, il ne donne que des indications sommaires, un peu surprenantes par aillcurs, sur le seul malade examiné. La sélection des patients n'a pas ćté non plus exempte de tout défaut puisque dans la séric de Yale, au moins, un patient présentant une affection différente du Parkinson a été inclus. Le diagnostic de la dégénérescence striato-nigrale dont il était atteint avait-il été fait avant la greffe (comme cela est probable) et, dans ce cas, qu'est-ce qui justifiait la procédure ? Enfin, et cela ne fait que renforcer lc sentiment de malaise ressenti face à ces deux publications, les deux équipes américaines ont introduit dans leurs protocoles de transplantation des modifications importantes dont l'utilité est loin d'avoir été démontrée chez l'animal. Ainsi, l'équipe de Redmond (Yale, CN, USA) [7] a utilisé systématiquement des tissus fotaux congelés, cryopréservés puis décongelés avant la transplantation. Les travaux expérimentaux que les auteurs sont presque seuls à avoir réalisés - en très petit nombre - ont démontré que, si des neurones vivants étaient récupérés, ils n'étaient plus capables de synthétiser qu'une faible fraction de la dopamine produite par des cellules-contrôle [8]. Le résultat négatif obtenu par cette équipe dans la recherche de neurones 
contenant la tyrosine hydroxylase, enzyme de synthèse des catécholamines, à l'autopsie d'un malade de leur série décédé quatre mois après la transplantation, est, de ce point de vue, assez inquiétant. Quant à la technique de dissection du tissu embryonnaire utilisée par l'équipe de Freed (Denver, CO, USA) [6], le moins que l'on puisse en dire c'est qu'elle est assez éloignée des protocoles jusque-là utilisés chez l'animal. Si le message "politique " transmis par les deux publications américaines est clair, puisqu'elles concourent à démontrer que des patients parkinsoniens pcuvent ĉtre améliorés par des transplantations intracérébrales de neurones fotaux, le message scientifique reste de ce fait, malheureusement, assez limité.

Les transplantations intracérébrales de neurones fotaux sont progressivement passées, au cours des trois dernières années, du laboratoire à la thérapeutique expérimentale contre la maladie de Parkinson. Les succès de cette approche sont probants. Il reste toutefois encore bien du chemin à parcourir pour que soit réellement évaluée la possibilité de les utiliser dans l'arsenal thérapeutique courant, comme le soulignent chacun de leur côté les auteurs des trois publications au New England Journal of Medicine. Pour que ce chemin soit parcouru, il faut vraisemblablement que l'aspect scientifique retrouve enfin tous ses droits et que la difficulté à faire admettre une nouvelle approche sur un plan éthique ne se traduise pas par un affaiblissement de la rigueur scientifique

\section{Marc Peschanski}

Directew de recherche à l'Inserm. CJF 91,02, neuroplasticité et greffes intracérébrales, centre hospitalo-universitaire Henni-Mondor, 51, avenue du Maréchal-de-Lattre-de-Tassigny, 94010 Crétal, France.

\section{TIRÉS A PART}

M. Peschanski

\section{RÉFÉRENCES}

1. Kassirer JP, Angell M. The use of fetal tissuc in research on Parkinson's discase. $N$ Engl J Med 1992 ; 327 : 1591-2.

2. Lindvall $\mathrm{O}$, Brundin $\mathrm{P}$, Widner $\mathrm{H}$, et al. Grafts of fetal dopamine neurons survive and improve motor function in Parkinson's discasc. Science 1990 ; 247 : 574-7.

3. Frecd CR, Brceze RE, Rosenberg NL, et al. Grafts of human fetal dopamine cells for Parkinson's diseasc : results at 1 ycar. Arch Neurol 1990 ; 47 : 505-12.

4. Lindvall O, Widner H, Rehncrona S, et al. Transplantation of fetal dopamine ncurons in Parkinson's disease : onc-year clinical and ncurophysiological obscrvations in two paticnts with putaminal implants. Ann Neurol $1992 ; 31$ : 155-65.

5. Widner H, Tetrud J, Rehncrona S, et al. Bilateral fetal mesencephalic grafting in two patients with parkinsonism induced by 1-mcthyl-4-phenyl-1,2,3,6-tctrahydropyridinc (MPTP). N Engl J Mad 1992 ; 327 : 1556-63. 6. Freed CR, Brecze RE, Rosenberg NE, et al. Survival of implanted fetal dopamine cclls and neurologic improvement 12 to 46 months after transplantation for Parkinson's diseasc. N Engl J Med 1992 ; 327 : 1549-55

7. Spencer DD, Robbins RJ, Naftolin F, et al. Unilateral transplantation of human fetal mesencephalic tissue into the caudate nucleus of patients with Parkinson's discasc. $N$ Engl $J$ Med 1992 ; 327 : 1541-8

8. Redmond DE, Naftolin F, Collier TJ, et al. Cryoprescrvation, culture and transplantation of human fetal mesencephalic tissuc into monkcys. Science 1988 ; 242 : 768-71. 